\title{
A haematological study of patients receiving long-term treatment with trimethoprim and sulphonamide
}

\author{
G. C. JENKINS, D. T. D. HUGHES, AND P. C. HALL \\ From The London Hospital, London
}

SYNOPSIS The haematological effects of long-term treatment for three months with a combination of trimethoprim and sulphamethoxazole were studied in 10 cases of chronic bronchitis. Trimethoprim selectively inhibits dihydrofolate reductase in bacteria but not in man. Only minor effects on folate metabolism were observed in nine cases, causing no clinical concern. Some degree of thrombocytopenia occurred in the tenth case which improved when folinic acid was given.

Trimethoprim, 2,4-diamino-5-(3'-4'-5'-trimethoxy benzyl) pyrimidine is an inhibitor of dihydrofolate reductase. It has an antibacterial activity which has been established since it was first described by Roth, Falco, Hitchings, and Bushby (1962) and its use in this field is gaining in popularity with the advent of more recent studies (Drew, Hughes, Fowle, and Cassell, 1967a; Darrell, Garrod, and Waterworth, 1968).

In bacteria trimethoprim prevents the reduction of dihydrofolate to tetrahydrofolate. In the earlier stage of the same metabolic pathway the conversion of para-amino-benzoic acid to dihydrofolic acid can be inhibited by sulphonamides. It is the synergic action of a combination of these drugs which has made it such an effective antibacterial agent. In view of the possibility that the drug combination might impair folate metabolism in man, we have studied the haematological effects during a long-term course of treatment in patients with chronic chest disease.

The 10 patients studied had an average age of 60.9 years. Seven had chronic bronchitis and three bronchiectasis. Each was given trimethoprim $250 \mathrm{mg}$ and sulphamethoxazole $500 \mathrm{mg}$ twice daily for three months, the ratio being 1:2. In addition to the clinical and bacteriological assessment, haematological investigations were made on the peripheral blood, bone marrow, and folic acid metabolism at intervals of two to three weeks (see methods). The subjects received a five-day course of treatment in hospital after Received for publication 11 September 1969. which repeat blood counts were performed; they were then allowed home and assessed as outpatients at intervals of one to two weeks. This study has already been briefly reported. (Drew, Hughes, and Jenkins, 1967b).

\section{Methods}

The methods employed for estimating serial haemoglobin $(\mathrm{Hb})$, packed cell volume (PCV), mean corpuscular haemoglobin concentration (MCHC), total white count (TWC), differential white cell counts and direct antiglobulin tests were essentially those described in 'Practical haematology' (Dacie and Lewis, 1968). Platelets were counted in duplicate on each occasion using a Coulter model A particle counter. The polymorphonuclear lobe counts were estimated by the method described by Herbert (1962) and the results expressed in lobe averages, that is to say, the total number of lobes in 100 neutrophils divided by 100 . Serum $L$. casei folate activity was estimated essentially by the technique of Spray (1964) though necessarily a specially modified culture of Lactobacillus casei resistant to the combination under study was employed ${ }^{1}$. Red cell folate activity in case 10 was assayed by the method described by Hoffbrand, Newcombe, and Mollin (1966) using

${ }^{1}$ Kindly supplied by J. Birchall of Burroughs Wellcome, Tuckahoe, N.Y. 


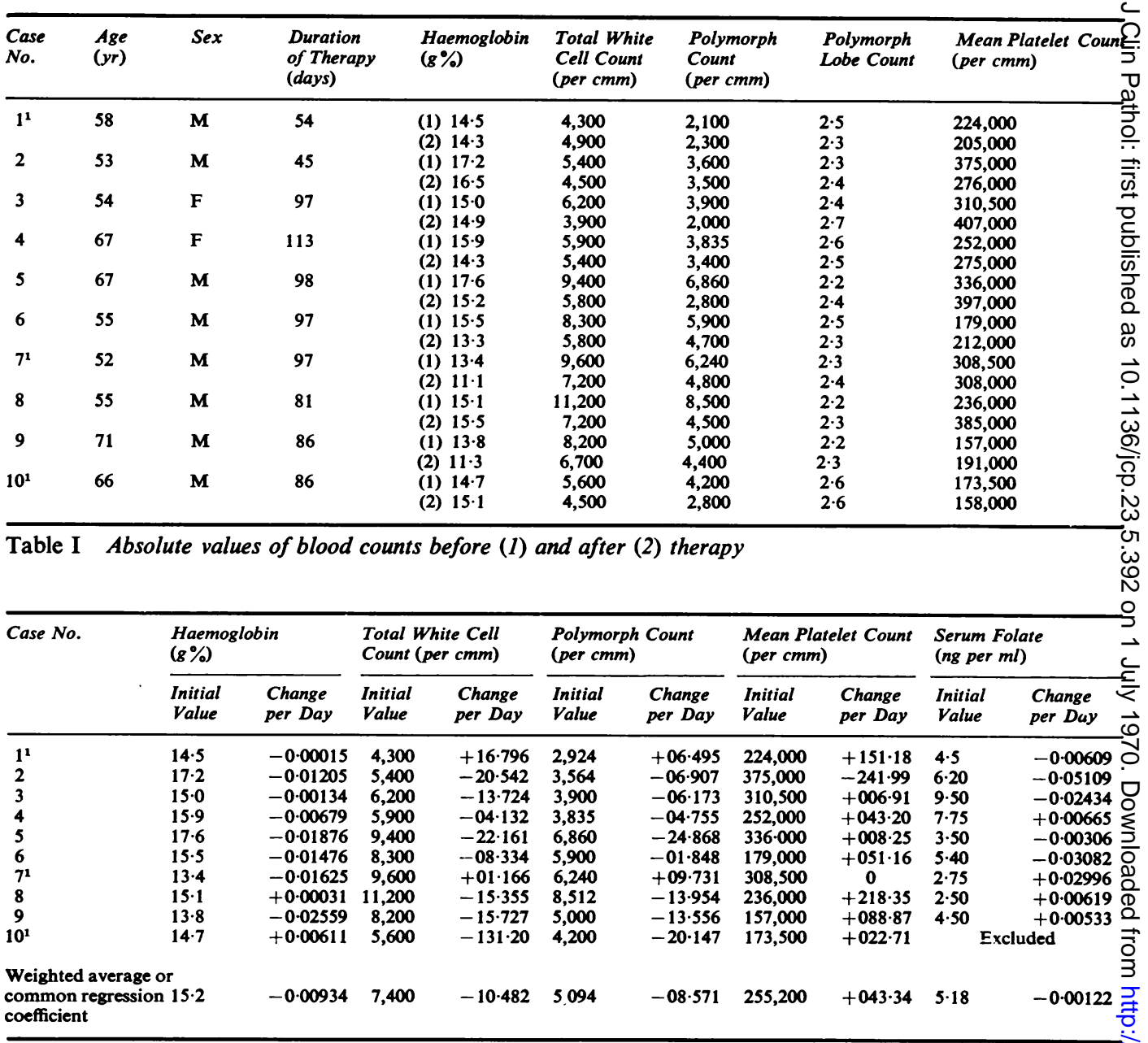

Table II Haematological values before treatment and estimates of the change per day during the course of therapy

II arriving at the weighted average or common regression coefficients all the values of the different parameters measured during the period of treatment were taken into account.

the same modified strain of $L$. casei. Urocanic acid excretion after a $15 \mathrm{~g}$ histidine load was measured by the method of Chanarin and Bennett (1961).

\section{Results}

Haematological data obtained on the cases before and after treatment is produced in Table I, which simply gives the values before and after therapy. When all the blood counts carried out during the period of treatment (up to 15 in some cases) are considered it is possible to estimate the expected daily change and common regression coefficient. These fuller results are shown in Table II and represent a more precise measurement of the changes produced, since much more data are considered. The changes can be summarized as follows:
HAEMOGLOBIN LEVELS, TOTAL LEUCOCYTE COUNTS, AND NEUTROPHIL COUNTS

There was a consistent small, but significant decrease in these values in eight subjects. Ther magnitude of these changes is depicted in Table IIN In all cases the haemoglobin values and neutro+ phil counts rose upon discontinuing treatment.

POLYMORPHONUCLEAR LOBE COUNTS Overall there was no significant increase in lobe counts at the end of treatment. In case 5 there was evidence of hypersegmentation of the nuclei, on three occasions when $20 \%$ of the cells had four or five lobes 68 to 84 days after starting treatment.

PLATELETS

Case 2 showed the only fall in number, all the other cases having a higher count after therapy 


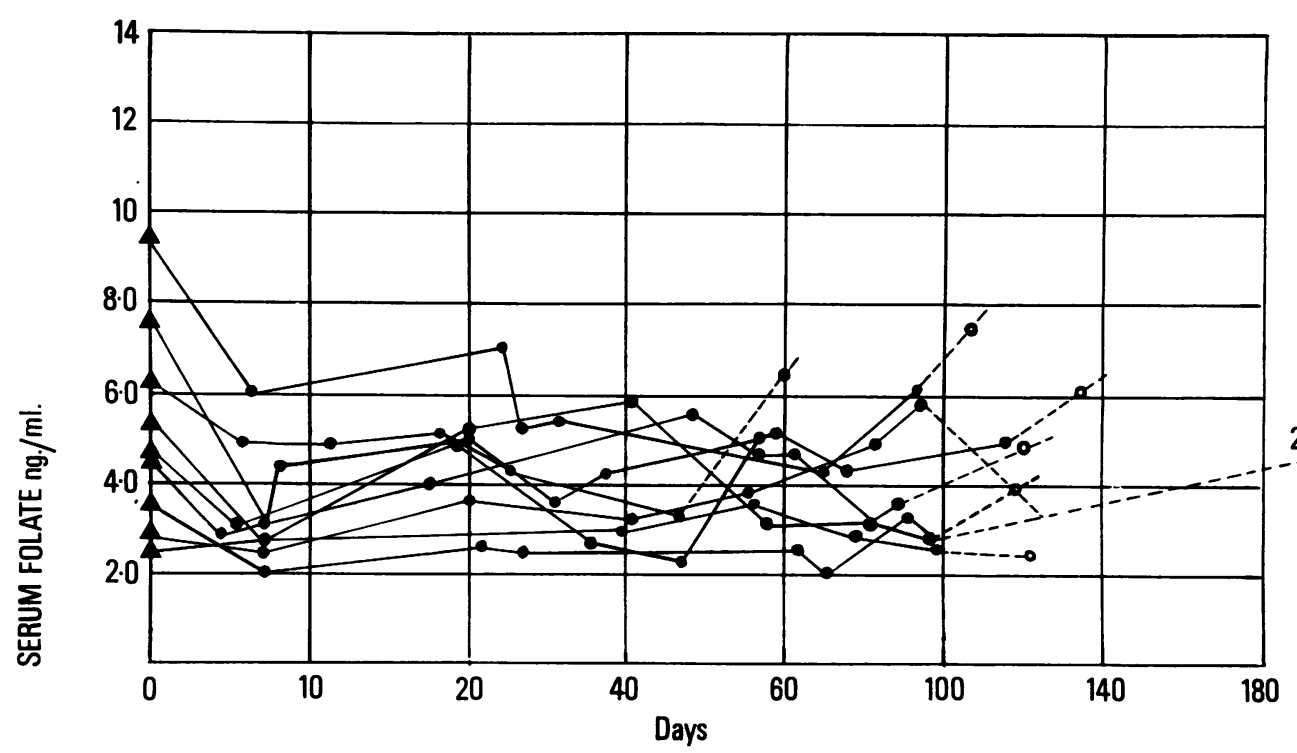

$\longrightarrow$ During therapy with Tmp/Smz

--- - - After therapy ceased

Fig. 1. Serial serum folate levels in nine cases.

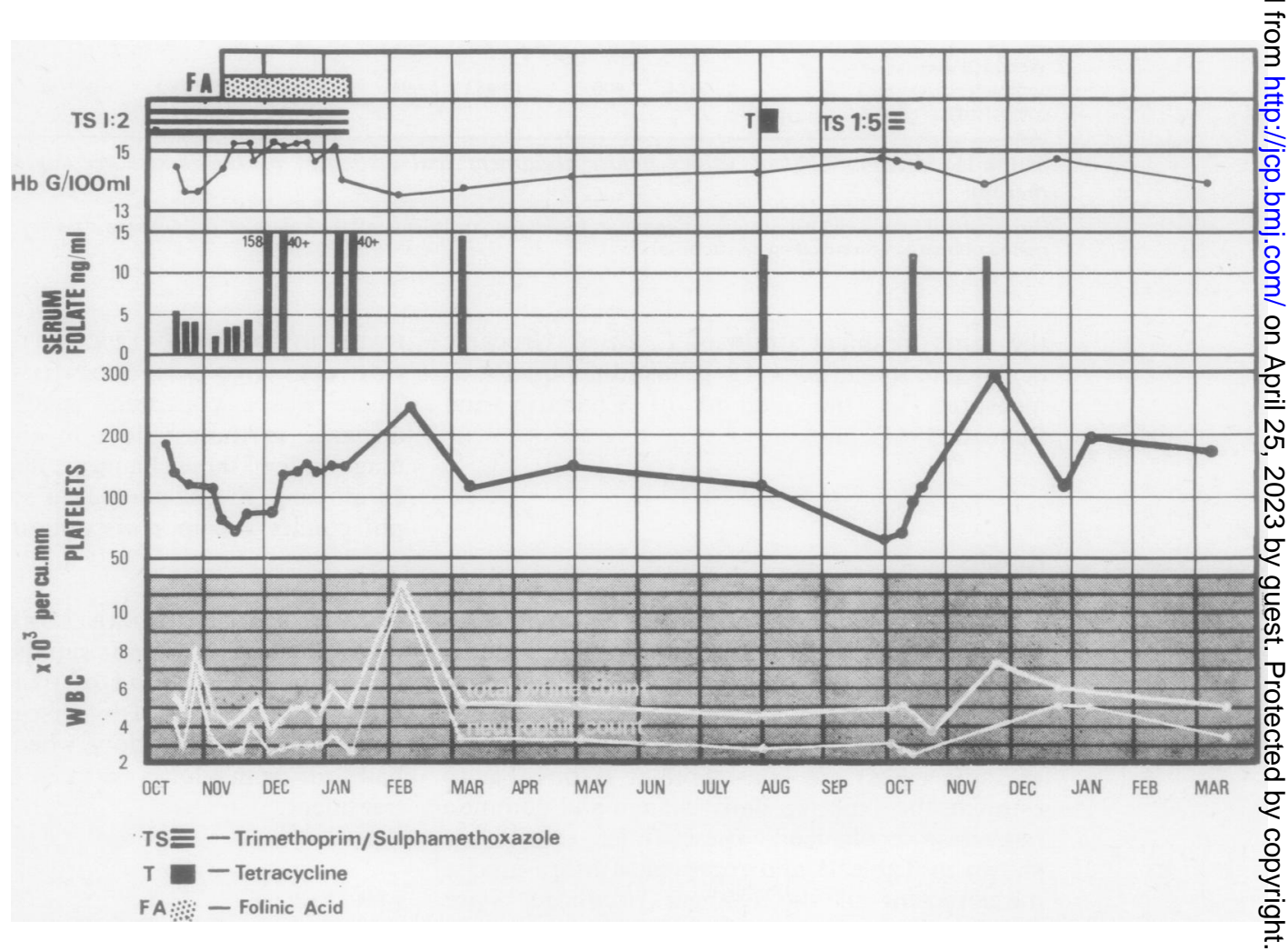

Fig. 2. Haematological data (case 10.) 
than before except in one case where the counts remained unchanged.

\section{SERUM L. CASEI FOLATE ACTIVITY}

Serial observations on nine of the cases in the series are depicted in Fig. 1 and the level at the beginning of treatment and the estimated daily change are shown in Table II. One subject (case 8) started with a near abnormally low level of activity (normal range in our laboratory $=2.4$ to $15 \mathrm{ng} / \mathrm{ml}$ ) and produced much the same value at the end of treatment. Cases 1 and 2 also had similar folate activities before and after therapy. The remaining cases showed varying small changes. A particular feature was the rapid fall in the first five days of treatment except in cases 7 and 8 , both of which started at low levels. This initial fall recovered within three weeks without any alteration in therapy and continued at an intermediate level until the course was completed (see Fig. 1). Only one case (no. 5) fell just below the lower limit of normal during treatment; at this time the neutrophil lobe counts were increased. In most cases the level rose again after therapy ceased. Urocanic acid measurements at the fifth day of treatment, when folate levels were in most cases at their lowest, showed no abnormal excretion. In all these cases the bone marrow pictures were normal in cellularity and cell morphology before and after treatment.

The tenth case in the series requires to be considered separately because folinic acid was administered during the course of treatment (Fig. 2).

\section{Case 10}

The subject was a 66-year-old man whose nutritional standard was not high. Haemoglobin remained high throughout his course of treatment, lasting for 86 days. From the beginning, however, the platelet numbers diminished until they reached 66,000 per $\mathrm{cmm}$ after 34 days' treatment. This downward trend was accompanied by a series of low serum folate values and a reduction in the absolute neutrophil count from 4,200 to 2,400 per $\mathrm{cmm}$. At this time the polymorphonuclear lobe count rose to its highest index of 3.14 and the presence of giant metamyelocytes was noted in the film of marrow aspirated together with some macronormoblasts.

In view of these findings calcium folinate was administered at first parenterally $(9 \mathrm{mg}$ intravenously) and later orally (5-15 $\mathrm{mg}$ daily) until the end of the three-month study. This was not detrimental to the clinical effects of the antibacterial therapy and was associated with a gradual rise in platelet count. It was not accompanied by any consistent rise in the neutrophil count. The marrow picture was then found to be normal.
Nearly nine months after the end of treatment $\bar{c}$ with both folinic acid and the combination, the circulating platelets were again at the low levelo which had given rise to concern during treatment. Despite these figures, for clinical reasons, the combination was readministered in the proportion of trimethoprim/sulphamethoxazole 1:5 for five? days after which the serum folate level wase normal $(7 \cdot 3 \mathrm{ng} / \mathrm{ml})$. Subsequent folate estimations were very similar and red celf folate estimations carried out two months later were well within the normal range, indicatinges that there was no tissue depletion of the vitamin at that time. Platelet counts showed a consistentrise in numbers immediately after this second $\vec{\omega}$ short course of treatment with the combination.

\section{Discussion}

In assessing the haematological effects of this $\mathrm{O}$ combination it is necessary also to consider the chronic infection for which these patients were being treated and its response to therapy.All of these cases responded clinically and both $\varphi$ the quantity and purulence of their sputum was. reduced (Drew et al, 1967b).

There is little doubt that in the high dosage used for this trial over a prolonged length of time there was some measurable decrease in available folate. Of interest is the early fall in $\stackrel{\varnothing}{\AA}$ serum folate occurring in the majority of cases $\vec{F}$ within the first week which was followed by aㄱ spontaneous rise in the following week or so.? A likely explanation of this is a partial overcomingo of the inhibition of dihydrofolate reductase in the tissues along the lines suggested by Bertino,? Donohue, Simmons, Gabrio, Silber, and Huen- $\frac{3}{3}$ nekens (1963). Using other powerful inhibitors of dihydrofolate reductase, such as amethopterin, $\frac{}{3}$ these workers postulated that inhibition of the enzyme resulted in decrease in the formation 9 of tetrahydrofolate with a resulting decrease in the synthesis of some metabolite that indirectly repressed the synthesis of dihydrofolate reductase. N Thus the enzyme level in the mammalian tissues $N$ tended to recover again after an interval of time. $N$ In describing the megaloblastosis induced by ${ }_{\sigma}^{\omega}$ the antimalarial agent, pyrimethamine, which is a drug closely related to trimethoprim chemically,, Waxman and Herbert (1969) demonstrated that this was an antifolate effect which could ${ }^{+}$ be completely reversed by giving folinic acid, $\frac{0}{0}$ but only poorly by folic acid.

Herbert (1965) considered that the earliest $\frac{?}{\Phi}$ biochemical abnormality in the development of $\varrho$ folate deficiency was a fall in serum folate level, so that measurement of this level was the most 8 sensitive biochemical index of a deficiency. Despite this detectable fall in serum folate, the magnitude of the haematological effect was small. The haemoglobin levels and neutrophil 
counts were consistently decreased but the neutrophil count would only have been decreased by 857 over a 100-day period. A proportion of this effect may be attributable to the sulphonamide. The only significant degree of hypersegmentation of the polymorphonuclear cells occurred in cases 5 and 10, patients who were in a poor nutritional state.

Using a dose of trimethoprim two times greater than in the present series, Kahn, Fein, and Brodsky (1968) found more evidence of inhibition of folate metabolism in man. In one series in which the trimethoprim alone was given for four weeks, two out of the 10 patients showed haematological toxicity in the form of thrombocytopenia and anaemia respectively, and five were described as having transitional megaloblastic erythroid changes in the bone marrow. In a long-term study in which trimethoprim $1 \mathrm{~g}$ was combined with sulphamethoxazole $4 \mathrm{~g}$ daily, 13 patients were given the combination for periods of six months to one year; haematological toxicity in the form of thrombocytopenia and neutropenia with raised lobe counts was ascribed to trimethoprim in two of these cases. In one case the administration of folinic acid reversed the adverse effects during the continuation of the drug. In our series, platelet counts tended to increase in number (Table II). In the first nine cases only one platelet count showed a fall. It is possible that the removal of the inhibiting effect on the bone marrow of a chronic infective state may account for this.

Like Kahn and his co-workers (1968), one case (no. 10) in our series showed the effects of folate deficiency in the first month of treatment. Apart from a series of low serum folate values this was also manifested by significant thrombocytopenia, neutropenia, and hypersegmentation of the polymorphonuclears. Administration of folinic acid rather than folic acid seemed logical so that tetrahydrofolic acid would be more readily available. As in the case described by Kahn et al (1968), a rise in platelet count occurred and the previously abnormal marrow picture returned to normal. However, it seems unlikely that the changes were entirely due to the effects of trimethoprim since the platelet level fell to low levels again nine months after stopping trimethoprim/sulphamethoxazole treatment at a time when serum and red cell folate levels were normal. Furthermore, a subsequent short course of the combined drugs was followed by a rise in platelet counts. Edwards, Jewkes, and Grant $(1970)^{1}$ also described a case receiving long-term trimethoprim-sulphamethoxazole treatment with evidence of folate deficiency. They demonstrated that these side effects failed to respond to therapeutic folic acid but that the administration of folinic acid produced a rapid improvement. These findings seem good evidence that in their case the abnormal haematology was due to an inhibitor of dihydrofolate reductase, that is to say, trimethoprim.

In our case (no. 10) the subject was in a poof nutritional state so that dietary deficiency may. have been an additional factor. The overall: drug doses used in this survey were considerably higher than those used in current clinical practice (preparations marketed at present contain trimethoprim $80 \mathrm{mg}$ with sulphamethoxazole $400 \mathrm{mg}$ per tablet, and a course of two tablet $\curvearrowright$ twice daily for seven days is recommended) Even so, the effect on human folate metabolism 5 was small and reverted to normal after cessationof therapy.

\section{References}

Bertino, J. R., Donohue, D. M Simmons, B., Gabrio, Silber, R., and Huennekens, F. M. (1963). The "induction of dihydrofolic reductase activity in leukocytes and ery $\mathrm{N}$ throcytes of patients treated with amethopterin. J. clin? Invest., 42, 466-475.

Chanarin, I., and Bennett, M. C. (1962). A spectrophotometricmethod for estimating formimino-glutamic and urocanic acid. Brit. med.J., 1, 27-29.

Dacie, J. V., and Lewis, S. M. (1968). Practical Haematology, 4th edition. Churchill, London.

Darrell, J. H., Garrod, L. P., and Waterworth, P. M. (1968) Trimethoprim: laboratory and clinical studies. J. clin: Path., 21, 202-209.

Drew, C. D. M., Hughes, D. T. D., Fowle, A. S. E., and Cassell M. A. (1967a). In Proceedings of Vth International Congress of Chemotherapy, vol. 1, p. 293.

Drew, C. D. M., Hughes, D. T. D., and Jenkins, G. C. (1967b) 이 In Proceedings of Vth International Congress of Chemo응 therapy, Suppl. (A 1-5a/3), p. 107.

Herbert, V. (1962). Experimental nutritional folate deficiency in man. Trans. Ass. Amer. Phycns, 75, 307-320.

Herbert, V. (1965). Folic acid. Ann. Rev. Med., 16, 359-370.

Hoffbrand, A. V., Newcombe, B. F. A., and Mollin, D. L. (1966) Method of assay of red cell folate activity and the value of the assay as a test for folate deficiency. J. clin. Path., 19, 17-28.

Kahn, S. B., Fein, S. A., and Brodsky, I. (1968). Effects of tri응 methoprim on folate metabolism in man. Clin. Pharm and Ther., 9, 550-560.

Roth, B., Falco, E. A., Hitchings, G. H., and Bushby, S. R. M (1962). 5-Benzyl-2,4,-diaminopyrimidines as antibacterial agents. I. Synthesis and antibacterial activity in vitro.3 J.med. Pharm. Chem., 5, 1103-1123.

Spray, G. H. (1964). Microbiological assay of folic acid activity음 in human serum. J. clin. Path., 17, 660-665.

Waxman, S., and Herbert, V. (1969). Mechanism of pyrimetha mine-induced megaloblastosis in human bone marrow. New Engl. J. Med., 280. 1316-1319. 